\title{
MULTILEVEL OPTIMIZATION OF GRILLAGES
}

\author{
Rimantas Belevičius ${ }^{1}$, Saulius Valentinavičius ${ }^{2}$, Edvard Michnevič ${ }^{3}$ \\ 1,3 Dept of Engineering Mechanics. ${ }^{2}$ Dept of Information Technologies, \\ Vilinius Gediminas Technical University, Sauletekio al. 11, LT-2040 Vilnius, Lithuania \\ ${ }^{1}$ E-mail:Rimantas.Belevicius@fm.vtu.lt2E-mail:S.Valentinavicius@Matrix-Software.lt \\ ${ }_{3}^{3}$ E-mail: edmich@fm.vtu.lt
}

Received 15 Octob 2001; accepted 21 Febr 2002

\begin{abstract}
The mathematical models and solution algorithms for optimization of grillage-type foundations are presented. Optimization of grillage is based on optimization of separate beams comprising grillage. Minimising of maximum in absolute value vertical reactive force, bending moment, and reaction-bending moment together is sought in a separate beam. All these problems are non-linear, therefore are solved iteratively changing in each iteration the structure shape to a better neighbouring shape. Solution of this requires three steps: finite element analysis, analytical sensitivity analysis, and optimal re-design via linear mathematical programming. The main problem related to the proposed technique is to guarantee the global minimum solution. Engineering algorithm is suggested for avoidance of local minimum solutions: optimization procedure starts from quasi-optimal initial pile placement scheme, which is designed by a special expert system.
\end{abstract}

Keywords: grillages, support reactions, bending moments, multilevel optimization, finite element method, analytical sensitivity analysis.

\section{Introduction}

Optimization is an inherent part of all engineering practice. In the construction of buildings means that, all parts of buildings from foundations to roofs should be designed and built optimally and thrifty as much as the conditions of safety and comfort allow. In this paper we shall concentrate on the optimal design of grillagetype foundations, which are the most popular and effective scheme of foundations, especially in case of weak grounds.

A grillage consists of separate beams. A separate beam may a) be supported by piles, b) reside on other beams; a mixed scheme may be the case. The optimal scheme of grillage should possess, depending on given carrying capacities of piles, the minimum possible number of piles. Theoretically, reactive forces in all piles should approach the limit magnitudes of reactions for those piles. Limit pile reactions may differ from beam to beam provided different characteristics (ie, diameters, lengths, profiles) of piles are given, or the piles are the same but the ground differs remarkably under different parts of building. Practically, this is difficult to achieve if designer, due to some considerations, introduces into the grillage scheme the so-called 'immovable supports' that have to retain their positions and do not participate in optimization process.
On the other hand, in order to optimally utilise the steel framework of joining beams, the bending moments in beams should be as small as possible and distributed uniformly over the structure, or, at worst, maximum positive moments should match the minimum ones.

Both goals can be achieved by choosing appropriate pile positions. Designer may arrive at the optimal pile placement scheme by engineering tests algorithms only in case of simple geometries and simple loadings. Otherwise mathematical optimization procedures are evident necessities. In this paper we try to pose mathematical optimization problems and to define solution methods, etc, up to the introduction into programme codes.

The inspiration for the work came from Dutch software company Matrix Software which produces and

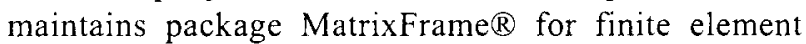
analysis and design of buildings. The package has built -in programme tool for optimal pile placement scheme based on engineering tests-and-errors approach. However, for complex grillages with thousands of piles it exposes unacceptable run-times or is unable to solve the problem correctly.

Here we describe completely different approach to the solution of aforementioned problems based on mathematical optimization. So far, excluding the authors' papers $[1,2]$, where the optimization of a single grillage beam was dealt with, any references were found in sci- 
entific literature. The single beam is optimized using 'move limit technique' [3-7].

\section{Idealizations}

Evidently both minimization problems of reactive forces in piles and bending moments in joining beam are non-linear and non-convex. If the whole grillage optimization problem is attacked, a) the complex mathematical apparatus should be involved, because pile positions have to follow the scheme of joining beams, which, in turn, is dependent on erection framework; b) global optimization is the must. Our choice is to attack problem from the simplest side, beginning with the optimization of separate beams and leaving aside graph theories and global optimization for future investigations.

Therefore basis for whole optimization process of grillage is the optimization of a single beam. All grillage is divided into separate beams, "the upper beams" resting on "the lower beams". First, all beams are analysed and optimised separately. Joints and intersections of the upper and lower beams are idealised as immovable supports for upper beams. Reactive forces in these fictitious supports are obtained during the analysis stage of the upper beams. Joints for the lower beams are idealised as concentrated loads of same magnitude but of opposite sign as reactive forces in fictitious supports. If more than 2 beams meet at joint, all beams are considered to be "the uppers" except for one the "lower". Distinguishing between the upper and lower beams can be done automatically by programme or by the designer's wish. Later on, as these fictitious reactions/concentrated loads depend on pile positions obtained in optimization problem, all calculations are embraced by iterative loop in order to level with proper accuracy forces at joints (or stiffnesses, if desired).

Now let us consider optimization of a particular beam of grillage. The specified data for optimization consist of:

- beam geometry

- beam material characteristics

- loadings (ie, concentrated loads and moments, distributed loadings of trapezoidal character)

- given immovable supports (if any). These supports will not participate in optimization process and will retain their positions

- fictitious immovable supports (if the beam is "upper")

- limit reactive forces in supports introduced through allowable reaction $R_{\text {allowable }}$ and factors to this reaction $f_{i}, i=1,2, \ldots$, number-of-movable-supports. This allows, in addition to multilevel optimization on whole grillage stage (different $R_{\text {allowable }}$ for different beams), a multilevel optimization on beam stage (different limit reactions for particular supports)

- minimum allowable distances between adjacent supports introduced purely due to technical conditions of pile erection process
All supports may be of two types: a) supports with specified displacements (where zero displacements are the most common case), and b) displacements with specified stiffness characteristics. First supports are rather nonrealistic representations and do not give satisfactory analysis results even in some simple cases. For example, when multiple supports are needed to carry large concentrated loads, this kind of supports will lead to a logjam. Provided odd number of supports is placed under load, and then central support will be located just beneath the load and will take all the force. In case of even number of supports the "saw-teeth" like distribution of reactions is observed, and the more supports will be installed, the larger in absolute value reactions will arise.

The problem has to be solved in statics and in linear stage. The problem size is up to 100 supports in separate beam and up to 150 beams in grillage, till 15 000 design parameters in total.

The only expected results are the least possible number of movable supports and those their positions by which all reactions do not exceed limit magnitudes, the bending moments are as small as possible and uniform.

\section{Problem statement}

Three optimization problems are to be examined: distribution of bending moments in joining beams, minimization of reactions at supports, and concurrent minimization of moments/reactions. The optimization problems are stated as follows:

Minimise (over feasible shapes) maximum $P$ (over beam and load cases)

with $P$ being the parameter to be optimised. The feasible shape of structure is defined by the type of certain supports, the given number and layout of different crosssections as well as different materials in the structure.

For the first problem parameter $P$ is maximum bending moment at some points of structure (eg, finite element mesh nodes):

$$
P=\left|M_{\max }\right| \text {. }
$$

The maximum difference between vertical reactive force at a support and allowable reaction for this support (introduced through given factors $f$ ) is used for the second problem, thus allowing to achieve different reactions at particular supports:

$$
\begin{gathered}
P=\left(R_{i}-f_{i}^{*} R_{\text {allowable }}\right)_{\text {max }}, \\
i=1,2, \ldots, \text { number-of- supports. }
\end{gathered}
$$

Minimization stops when $P_{\max }$ reaches zero magnitude.

Concurrent minimization of moments/reactions is more complicated. So far, any successful merit function for problem has been found, therefore a pseudoconcurrent optimization is performed using engineering algorithm:

1) Optimise reactions using $0.8^{*} R_{\text {allowable }}$ in merit function (2); when this problem is solved, go to 2 . 
2) Optimise moments using merit function (1). 2.1) If $P_{\max }>0$ return to 1.

If uniform distribution of moments is achieved, stop.

Clearly all problems are highly non-linear. Our choice is to use robust and reliable methods: finite element method for static analysis and linear mathematical programming (Simplex procedure) for optimization. Thus the problems have to be solved iteratively and are converted to a sequence of approximately linear problems of an optimal re-design. In each iteration the current shape is changed to a better neighbouring shape. The solution requires three steps:

- finite element analysis,

- sensitivity analysis with regard to design parameters,

- optimal re-design with linear programming.

Further, the minimum-maximum problem is converted to a pure minimum problem with constraints by treating $P_{\max }$ as unknown subject to constraints that $P_{\max }$ limits the magnitudes of parameter $P$ everywhere in the structure and for all load cases when design changes $\Delta t_{i}$ are performed

$$
P(x)+\sum_{i} P(x)_{{ }^{\prime} t_{i}} \Delta t_{i}-P_{\max } \leq 0
$$

for the total structural space $x$. The comma here and below means the differentiation.

For computational reasons a beam length constraint $L=\bar{L}$ is also included:

$$
L+\sum_{i} L_{t_{i}} \Delta t_{i}-L=0
$$

Several possibilities exist in the choice of design parameters $t_{i}$ on which the structure shape depends. Our choice is to use the most evident from the engineering point of view parameters: nodal co-ordinates of all (or a chosen set of) supports.

\section{Optimization technique and algorithm}

With reference to $[3,4]$, let us shortly describe the optimization procedures, first for a single beam and then for the whole grillage.

Optimization procedure for a single beam. Two absolute limits sets (maximum and minimum) on all design co-ordinates status $\mathbf{T}$ are introduced according to existing design restrictions or other considerations. In any case the design variable cannot exceed these limits. For the first solution step, current design variables status $\mathbf{T}=\mathbf{0}$. The absolute limits may differ from one design variable to another, however the maximum absolute move limits must be positive, but the minimum ones negative:

$$
\begin{aligned}
& T^{\max } \geq 0, \\
& T^{\min } \leq 0, \\
& T^{\min } \leq \mathrm{T} \leq \mathrm{T}^{\max } .
\end{aligned}
$$

Further, the move limits on the design variables al- terations $\Delta \mathbf{T}$ per one iteration are introduced, again maximum and minimum. These move limits may vary from one design variable to another and have to be adjusted to the extent of non-linearity of problem so that Simplex' predictions on the future behaviour of the structure do not differ remarkably from finite element solution. In general, move limits should be gradually shrunk as the design approaches the optimum. The accuracy of the approximation is required to be higher when we get close to the optimum because the gains are small and can be swamped by approximation errors. Adjustment of iteration move limits can be done easily by special programmed procedures. Thus,

$$
\Delta \mathbf{T}^{\mathrm{min}} \leq \Delta \mathrm{T} \leq \Delta \mathbf{T}^{\mathrm{max}} .
$$

Later on, a two sets of intermediate always positive variables $\Delta \mathbf{T}^{+}$and $\Delta \tilde{\mathbf{T}}$ are introduced in order to satisfy requirements of Simplex procedure. Omitting details (see $[1,2,3])$

$$
\Delta \mathbf{T}^{+}+\Delta \tilde{\mathbf{T}}=\Delta \mathrm{T}^{\max }-\Delta \mathrm{T}^{\min }
$$

Now the final problem formulation for mathematical programming is:

Minimise $P_{\max }$

with constraints:

level of $P$ everywhere in the structure $\leq P_{\max }$;

design changes do not exceed move limits, and design status does not exceed absolute limits;

length of model is constant.

Considering only the first derivatives in Taylor's expansion, the first constraints at the nodal points of structure become

$$
\mathbf{P}+[P]_{, \mathbf{T}} \Delta \mathbf{T}-\mathbf{P}_{\max } \leq \mathbf{0},
$$

or avoiding the inequality

$$
\begin{aligned}
{[I] \tilde{\mathbf{P}}-1 P_{\max }+[P]_{, \mathbf{T}} \Delta \mathrm{T}^{+}=} \\
-\mathbf{P}-[P]_{, \mathrm{T}} \Delta \mathbf{T}^{\min } .
\end{aligned}
$$

The second group of constraints in matrix notation for all design variables is

$$
\Delta T^{+}+\Delta \tilde{\mathbf{T}}=\Delta \mathbf{T}^{\max }-\Delta \mathrm{T}^{\min },
$$

while the third one is as follows:

$$
L+\sum\left[L^{e}\right], \mathbf{T} \Delta \mathbf{T}=\bar{L}
$$

where the sum covers only the active elements, ie including the current design variable as a node of element. In the first iteration $\mathrm{L}=\overline{\mathrm{L}}$.

Optimization procedure for grillage. First of all, "the upper" and "lower beams" are distinguished, either automatically or by designer. Optimization of separate beams then has to be embraced by accuracy loop because pile placement scheme of one beam influences reaction distributions in remaining beams. The following algorithm is employed: 
1) Starting accuracy loop. Initialisation: stiff-nesses at fictitious immovable supports of upper beams simulating joints with lower beams, accuracy tolerance.

1.1) Optimization of the upper beams using defined in the last iteration stiffnesses of fictitious immovable supports.

1.2) Optimization of the lower beams in addition to specified loadings taking into account concentrated loads coming from the upper beams.

1.3) If stiffnesses of the upper and lower beams at joints do not match (with specified accuracy), go to 1.1.

1.4) Filtering results to exclude matching supports at joints of beams. Stop.

\section{Finite element. Matrices for sensitivity analysis}

\section{Finite element matrices}

Simple two-node beam element with 4 d.o.f.'s [8, 9] has been implemented in analysis. Nodal d.o.f.'s of element are:

$$
\mathbf{u}=\left\{w_{i}, \theta_{i}, w_{j}, \theta_{j}\right\}^{T}
$$

$w_{k}$ and $\theta_{k}, k=i, j$ being deflection and rotation, positive counter-clockwise, accordingly.

The interpolation functions for all d.o.f.'s in Cartesian co-ordinates are as follows:

$$
[N]=\left\{\begin{array}{r}
1-\frac{3 x^{2}}{L^{2}}+\frac{2 x^{3}}{L^{3}} \\
x-\frac{2 x^{2}}{L}+\frac{x^{3}}{L^{2}} \\
\frac{3 x^{2}}{L^{2}}-\frac{2 x^{3}}{L^{3}} \\
-\frac{x^{2}}{L}+\frac{x^{3}}{L^{2}}
\end{array}\right\}
$$

with $L$ for the length of an element.

Bending moments at nodes, positive when cause the "positive" layers of a finite element experience tension, are related to deflections by flexural rigidity:

$$
M=-E I w_{x x}=E I \sum_{i} N_{i}, x x u_{i}
$$

After the nodal displacements are obtained, the reactive forces are available according to:

$$
R_{i}=\sum_{j}\left[\mathbf{K}_{i j}\right] \mathbf{u}_{j} .
$$

\section{Relations for sensitivity analysis}

As seen from (3), the sensitivity (ie, derivatives with regard to nodal co-ordinates) of bending moments and reactive forces is the must for optimization:

$$
\mathbf{M}_{x_{k}}=-E I \sum_{i}\left(N_{i, x \times x_{k}} u_{i}+N_{i, x x} u_{i, x_{k}}\right),
$$

$$
\mathbf{R}{, x_{k}}=[K]_{, x_{k}}^{a} \mathbf{u}^{a}+[K]^{a} \mathbf{u}_{, x_{k}}^{a}
$$

with superscript $\alpha$ standing for ensemble.

The derivatives of nodal displacements is obtained by solution of general sensitivity analysis

$$
[K]^{a} \mathbf{u}_{, x_{k}}^{a}=\overline{\mathbf{P}}^{a}
$$

with pseudo-load vector

$$
\overline{\mathbf{P}}^{a}=\mathbf{P}_{x_{k}}^{a}-[K]_{, x_{k}}^{a} \mathbf{u}^{a} .
$$

The procedure for derivative of element stiffness matrix from which matrix of ensemble $[K]^{a}, x_{k}$ is composed as follows: replace $L$ with $x_{j}-x_{i}$, detect whether $k$ is ith or $j$ th node of an element, and obtain $[K], x_{i}$ or $[K], x_{j}$, respectively. Thus only the element possessing node $\mathrm{k}$ renders non-zero stiffness derivatives.

Similar procedures are valid for derivatives of forces and reactions.

All matrix expressions are lengthy, therefore computer algebra was employed for them.

\section{Programme}

The finite element computational procedure, sensitivity analysis and optimal re-design via linear programming form the programme kernel (written in Fortran 90), which is supplemented with pre- and post-processing capabilities (in $\mathrm{C}++$ ). Natural desire of the designer is the "one button click" programme that automatically renders optimal solution. Main problem of suggested technique is caused by the use of linear mathematical programming. Except for problems with relatively simple geometry and loading, it inevitably leads to a local minimum solution. However, it is hardly conceivable to use procedures of global optimization for problems of a given size [10-12]. Evident way out of situation is to start optimization procedure from near-optimum initial scheme. Special expert system is created which analyses geometry of beam, loadings, carrying capacities of piles and yields quasi-optimal initial pile placement scheme. Initial finite element mesh is prepared automatically, introducing nodes at support places, jumps of material and cross-sections properties, etc. Quite dense finite element mesh is necessary, primarily due to the only evaluation procedure of bending moments at calculated via arithmetic mean of bending moments obtained from neighbouring finite elements, and this makes the moment derivatives more sensitive to the finite element lengths.

The pre-processor allows the supports - "master nodes", ie, nodes co-ordinates of which are design variables in optimization procedure, to move over structure freely following Simplex solutions. The only exception is situation when two or more master nodes meet: in this case the programme suspends them at given minimum allowed distance between adjacent supports. This may cause small numerical disturbances in sensitivity analysis. 

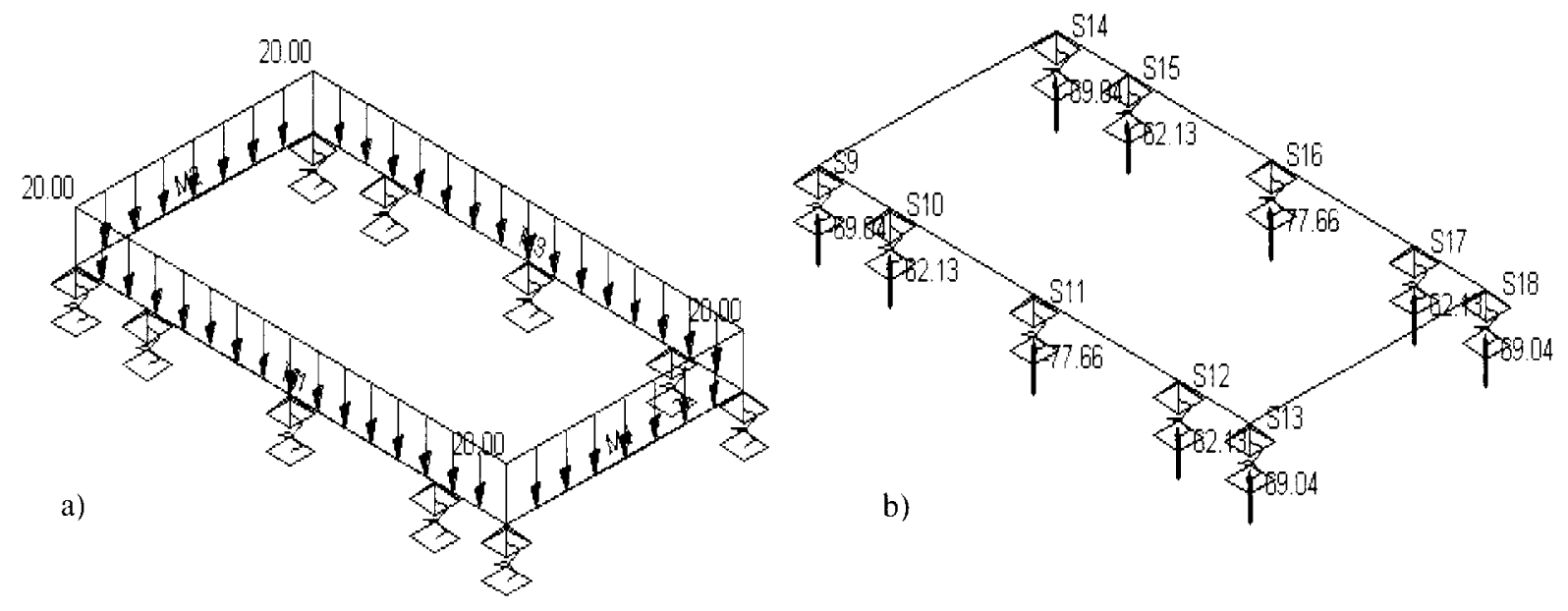

c)

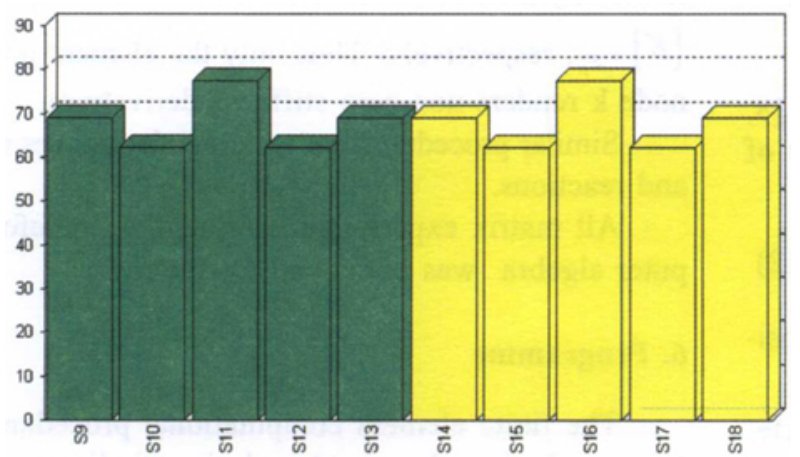

Fig 1. Supports placement optimization of grillage with spring type supports. a) Loading of structure. b) Support reactions after optimization. c) Optimization quality histogram.

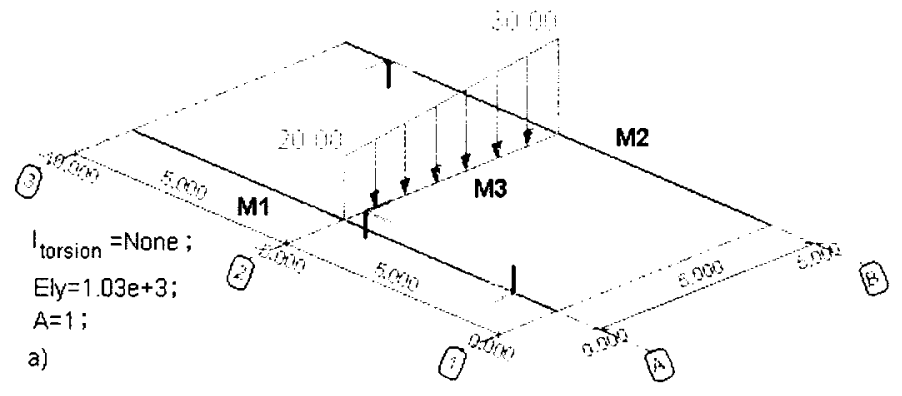

b)

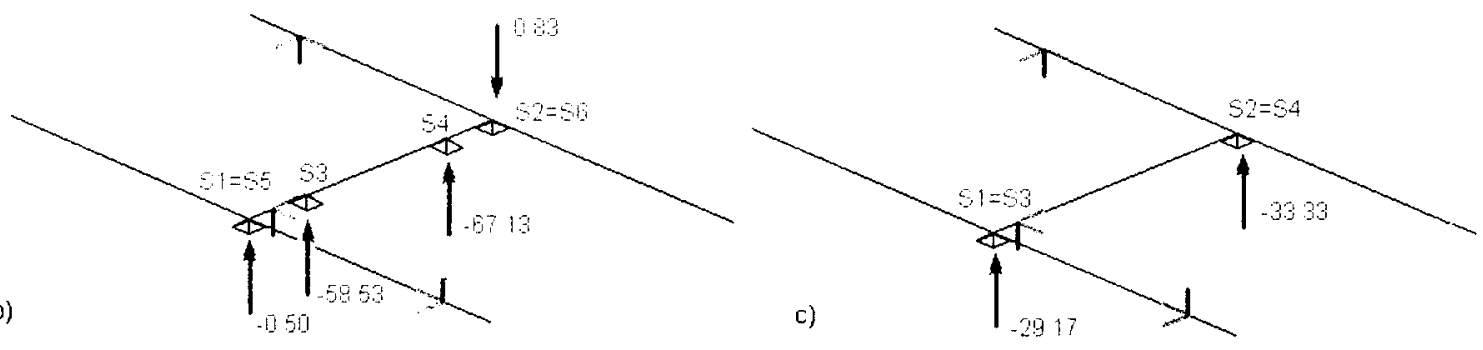

Fig 2. Optimization of grillage with the fixed supports type. a) Geometry and loading of structure. b) Optimization results when shear forces redistribution factor is $1 \%$. c) Optimization results when shear forces redistribution factor is $100 \%$ 


\section{Numerical examples}

The following numerical examples demonstrate the capabilities of the proposed technique. We restrict ourselves with examples of reaction minimisation of academic character in order to be able to compare the obtained results with the optimal shapes of grillages known in advance.

Example 1. Grillage of rectangular shape loaded with uniformly distributed loading of intensity 20. All the supports should be generated by programme; supports with specified vertical stiffness $\mathrm{k}_{\text {spring }}=10^{6}$ were given for programme, while $\mathrm{R}_{\text {allowable }}=100$. The programme yields 10 spring-supports placed under "the lower", longer beams. The required tolerance of calculations is achieved in 3 iterations of accuracy loop. Note, the reactions in supports are not equal (Fig 1, c), because the programme stops optimization loops after the allowable reaction is not exceeded in any support.

Example 2. Grillage with supports with specified zero displacements (Fig 2). Central beam rests on two "lower beams". This purely academic example demonstrates another mechanism for loading transmission from the upper beam to the lower ones. If due to some considerations the user does not want the loading from the upper beam to be transmitted to the lower ones, he introduces small "shear redistribution factor" (say, of value $1 \%$ ) for "the upper beam". In this case almost all loading is taken by supports placed under the upper beam (Fig $2 \mathrm{~b}$ ). This is achieved by multiplying the magnitude of allowable reaction for immovable supports at the ends of upper beam by given "shear redistribution factor". These immovable supports simulate the influence of lower beams to the behaviour of upper beam. Now the upper beam is optimised using 2 sets of allowable reactions: one for movable supports inside beam and second for immovable supports.

Otherwise, in case of factor magnitude $\geq 100 \%$, loading through joints goes to the lower beams and is taken by supports placed under the lower beams.

\section{Concluding remarks}

The mathematical models for optimization of grillage-type foundations are presented. Minimising of maximum in absolute value vertical reactive force, bending moment, and reaction-bending moment together is sought. Optimization of grillage is based on optimization of separate beams comprising grillage. Finite element method, analytical sensitivity analysis and linear mathematical optimization are employed in the proposed technique.
Engineering algorithm is suggested for avoidance of local minimum solutions: optimization procedure starts from quasi-optimal initial pile placement scheme, which is designed by special expert system. Solutions of a number of problems demonstrate the validity of proposed algorithms. New investigations of merit functions are needed for the case of joint optimization of reactions and moments. Investigations of possibilities to use global optimization and graph theories in grillage optimization are foreseen in the future.

\section{References}

I. Belevičius R., Valentinavičius S. Optimization of grillagetype foundations. Civil Engineering (Statyba), Vol 6, No 6, 2000 , p. $255-261$

2. Belevičius R., Valentinavičius S. Optimization problems for foundations of erections. Lithuanian Mathematical Collection, Vol 40, 2000, p. 365-373.

3. Pedersen P. Design for minimum stress concentration some practical aspects. Structural Optimization, Kluwer Academic, 1988, p. 225-232.

4. Belevičius R. Shape optimization of laminated orthotropic plate structures. Mech. of Composite Mater., Vol 29, 1993. p. 537-546.

5. Pedersen P., Tobiesen L., Jensen S. H. Shapes of orthotropic plates for minimum energy concentration. Mechanics of Structures and Machines, Vol 20, No 4, 1992, p. 499514.

6. Pedersen P. On optimal shapes in materials and structures. Structural Optimization, Vol 19, 2000, p. 169-182.

7. Pedersen $\mathrm{P}$. On the influence of boundary conditions, Poisson's ratio and material non-linearity on the optimal shap. Int. J. of Solids and Structures, Vol 38, No 3, 2001, p. $465-477$

8. Cook R. D., Malkus D. S., Plesha M. E. Concepts and applications of finite element method. John Wiley \& Sons, 1989.

9. Zienkiewicz O. C., Taylor R. L. The Finite Element Method. $4^{\text {th }}$ ed., McGraw-Hill, 1991.

10. Horst R., Pardalos P. M. Handbook of Global Optimization. Kluwer Academic, 1995.

11. Kearfott R. B. Rigorous Global Search: Continuous Problems. Kluwer Academic, 1996.

12. Horst R., Pardalos P. M., Thoai N. V. Introduction to Global Optimization. $2^{\text {nd }}$ ed., Kluwer Academic, 2000.

\section{Acknowledgement}

Authors wish to thank the joint venture Matrix Software for inspiration, permission to use software package MatrixFrame ${ }^{\circledR}$ and financial support. 